\title{
Comparison of the success rate after debridement, antibiotics and implant retention (DAIR) for periprosthetic joint infection among patients with or without a sinus tract
}

Wang Deng ${ }^{1 \dagger}$, Rui $\mathrm{Li}^{2 \dagger}$, Hongyi Shao ${ }^{1}$, Baozhan Yu ${ }^{2,3}$, Jiying Chen ${ }^{2^{*}}$ and Yixin Zhou ${ }^{1 *}$

\begin{abstract}
Background: The relevance between the presence of a sinus tract and the failure risk after debridement, antibiotics and implant retention (DAIR) for periprosthetic joint infection (PJI) after hip or knee arthroplasty is still unclear. This study aimed to compare the success rate of DAIR between patients with or without a sinus tract and to explore the possible risk factors for failure after DAIR in patients with a sinus tract.

Methods: Consecutive DAIR cases for PJI after hip or knee arthroplasty between January 2009 and June 2019 with a minimum 1-year follow-up in two tertiary joint arthroplasty centers were included. Patients were classified into the sinus tract group and the non-sinus tract group according to the presence of a sinus tract. The success rate after DAIR were compared using Kaplan-Meier survival analysis. Potential risk factors for failure in the sinus group were also explored.
\end{abstract}

Results: One hundred seven patients were included. At a median 4.4years of follow-up, 19 of 52 (36.5\%) cases failed in the sinus tract group, while 15 of 55 (27.3\%) cases failed in the non-sinus tract group. The 1-year and 5-year cumulative success rates were $71.2 \%$ (95\% confidence interval (Cl): 59.8-84.6\%) and 56.8\% (95\% Cl: 42.6-75.7\%) in the sinus tract group, respectively, which were similar to the counterparts in the non-sinus tract group $(P=0.214)$. Among patients with a sinus tract, DAIR with the exchange of modular components showed a higher success rate (75.8\% versus $47.4 \%, P=0.038)$.

Conclusions: The presence of a sinus tract does not affect the success rate of DAIR. Modular component exchange in DAIR was proposed for patients with a sinus tract for an improved infection control rate.

Keywords: Debridement, antibiotics and implant retention (DAIR), Periprosthetic joint infection (PJI), Sinus tract

*Correspondence: chenjiying_301@163.com; orthoyixin@yahoo.com

†Wang Deng and Rui Li contributed equally to this manuscript and should be considered co-first authors.

${ }^{1}$ Department of Orthopedic Surgery, Beijing Jishuitan Hospital, Fourth Clinical College of Peking University, No.31 Xinjiekou East Road, Xicheng District, Beijing 100035, China

2 Department of Orthopaedics, Chinese PLA General Hospital, No. 28 Fuxing Road, Haidian District, Beijing 100039, China

Full list of author information is available at the end of the article

\section{Background}

Periprosthetic joint infection (PJI) is one of the most devastating complications after joint arthroplasty, with an incidence of $1-2 \%[1,2]$. The total number of PJI cases will increase due to the surge of new arthroplasties worldwide and previously implanted prostheses [2, 3]. Treatment of PJI brings great suffering to patients, poses permits use, sharing, adaptation, distribution and reproduction in any medium or format, as long as you give appropriate credit to the original author(s) and the source, provide a link to the Creative Commons licence, and indicate if changes were made. The images or other third party material in this article are included in the article's Creative Commons licence, unless indicated otherwise in a credit line to the material. If material is not included in the article's Creative Commons licence and your intended use is not permitted by statutory regulation or exceeds the permitted use, you will need to obtain permission directly from the copyright holder. To view a copy of this licence, visit http://creativecommons.org/licenses/by/4.0/. The Creative Commons Public Domain Dedication waiver (http://creativeco mmons.org/publicdomain/zero/1.0/) applies to the data made available in this article, unless otherwise stated in a credit line to the data. 
difficult challenges to surgeons and causes huge financial burdens $[4,5]$. The main treatment options involve debridement, antibiotics and implant retention (DAIR), one-stage and two-stage revision [6].

Compared with one-stage or two-stage revision, DAIR has the advantages of lower cost, lower skill requirement and better postoperative joint functions [7-9]. Numbers of cases treated with DAIR have increased rapidly in recent years $[6,10,11]$. However, the infection control rate after DAIR ranges from 10 to $100 \%$ in different studies, and the overall infection control rate in a recent meta-analysis is approximately $60 \%$ [12]. Identifying the possible risk factors for failure after DAIR is of great clinical significance since it may help to ensure higher infection control rate [13]. As an important sign of deep infection, a sinus tract could present in either acute or chronic infection [14-16]. However, data regarding the presence of a sinus tract were not reported in most studies that aimed to explore the risk factors for failure after DAIR [17-20]. Recent meta-analyses also did not mention this topic [12, 21, 22]. Patients with a sinus tract do go through DAIR to treat PJI in the clinic, and the relevance between the presence of a sinus tract and the failure risk after DAIR is still unclear [23-27]. To our knowledge, no studies have directly focused on the comparison of the infection control rate after DAIR for PJI after hip or knee arthroplasty between patients with or without a sinus tract, nor have any studies explored the possible risk factors for failure after DAIR in patients with a sinus tract.

The aims of this study were to (1) compare the PJI control rate after DAIR between patients with or without sinus tract and (2) explore the possible risk factors for failure after DAIR in patients with a sinus tract in a multicenter study.

\section{Methods}

\section{Patient inclusion}

Following Institutional Review Board approval, this study was performed with consecutive hip or knee PJI cases treated with DAIR from January 2009 to June 2019 in two tertiary joint arthroplasty centers. The inclusion criteria also involved (1) meeting the 2013 International Consensus Meeting (ICM) definition of PJI after hip or knee arthroplasty [28]; (2) DAIR under open arthrotomy; (3) first time surgical treatment for PJI and (4) at least one year of follow-up. The exclusion criteria were as follows: (1) superficial site infection and (2) index surgery using mega-prosthesis. Data on demography, joint surgical history and infection classifications were retrieved from the medical records. Acute infection was defined when the interval time from index surgery to DAIR was less than 4 weeks. If an abrupt onset of infection symptoms (e.g., fever, swelling, tenderness, wound drainage, etc.) occurred in a prior symptom-free prosthetic joint 4 weeks after the index surgery and the symptom duration from onset to DAIR was less than 3 weeks, the cases were defined as acute hematogenous PJI. Otherwise, the infection was considered chronic $[29,30]$. The sinus tract was defined and verified intraoperatively as a channel communicating the outside and the joint cavity [14]. All patients were divided into the sinus tract group and the non-sinus tract group according to the presence of a sinus tract.

\section{Treatment protocol}

All the included cases underwent DAIR by fellowshiptrained arthroplasty surgeons in two tertiary joint arthroplasty centers in our region. The two centers shared the same therapeutic protocol. With the definite or suspected diagnosis of PJI, a multidisciplinary team made the decision in favor of DAIR with the consent of the patient after fully understanding of all the treatment options, including one-stage or two-stage revision, DAIR or antibiotic suppressive treatments and their possible risks and benefits. A posterior lateral approach for hip and midline incision with a medial patellar approach for the knee was used for open arthrotomy. Sinus tracts were explored and excised roundly after confirmation of the communication with the joint cavity. Implants were assessed as well-fixed for DAIR; otherwise, one-stage or two-stage revisions were performed. After taking the samples for microbial cultivation and joint fluid analysis, radical synovectomy around the joints and excision of all the potentially infected tissue and sequestrum were performed. After thorough pulse lavage with antiseptic saline, 3\% hydrogen peroxide and diluted iodophor solutions were used as anti-infection agents for five minutes and were repeated at least twice during surgery. Modular components were removed for thorough debridement. Exchange to new modular components or reinsertion of the original ones was decided by the surgeons after considering the reusability of original ones and the availability of new ones. Utilization of topical antibiotics was also decided by the surgeons. Commonly, two grams vancomycin were chosen as the topical antibiotics for PJI caused by gram-positive bacteria or unknown pathogen while one gram meropenem was used for PJI with gramnegative bacteria.

If the microbial culture results were available before the debridement, antibiotics were chosen under the guidance of antibiotic susceptibility tests and advice from pharmacists. Vancomycin and a third-generation cephalosporin were combined to cover both gram-positive and gram-negative bacteria for patients without preoperative microbial culture results. Organism-specific 
antibiotics were administered when the intraoperative culture results were reported. For culture-negative cases, we maintained these broad-spectrum antibiotics [31]. Patients received intravenous antibiotics for at least two weeks and then oral antibiotics for at least another four weeks. During the hospitalization, clinical symptoms, such as swelling, redness, wound drainage, were checked when changing the wound dress. Infection biomarkers, including blood routine test, CRP and ESR, were tested every other day in the first week and once a week afterwards. If the clinical symptoms subsided and the infection biomarker decreased, we continued the wound care and antibiotic administration in accordance with the protocol. If the symptoms did not relive or the biomarker was still high or raised again after a drop, subsequent surgical intervention or continuous suppressive antibiotic therapy was decided by the multidisciplinary team and the patients. Postoperative functional recovery after DAIR began after removing the drainage tube. The discharging criteria included the relief of infection symptoms, the decrease and stabilization of infection biomarkers. The total duration of antibiotics was between 6 to 12 weeks [32].

\section{Follow-up and outcome assessment}

After being discharged from the hospitals, we encouraged patients to attend clinic visits routinely and to return to the hospital as soon as possible if any infection symptoms occurred. The success of DAIR was defined according to the definition suggested by Diaz-Ledezma et al.: (1) infection eradication, characterized by a healed wound without sinus tracts, drainage, or pain, and no infection recurrence caused by the same organism strain; (2) no subsequent surgical intervention for infection after DAIR; and (3) no occurrence of PJI-related mortality (by causes such as sepsis and necrotizing fasciitis) [33]. Cases with chronic infection symptoms and treated with antibiotic suppressive therapy were defined as failure. If the DAIR was defined as failed, we recorded the time of failure, subsequent treatment options and the status of the joint at the last follow-up.

\section{Statistical analysis}

In the comparison between the sinus tract and nonsinus tract groups, continuous variables in demographic data were expressed as the mean with range or the median with interquartile range (IQR) and were compared by Student's T-test or Mann-Whitney U test according to the Kolmogorov-Smirnov test of normality. Categorical variables were compared between two groups using Chi-square test or Fisher's exact test. The DAIR outcomes were depicted and compared between the two groups using a Kaplan-Meier survival analysis (log-rank test), and the cumulative 1-year and 5-year infection control rates were reported. Among patients with a sinus tract, possible risk factors were also compared between the successful and failed groups. Kaplan-Meier survival analysis was also performed for the potential risk factors. The significance level was set at a $P$-value $<0.05$.

\section{Results}

One hundred eighteen patients were reviewed initially, and 107 cases were included after excluding 9 patients for superficial site infection and 2 for mega-prosthesis. Fifty-two patients showed the presence of a sinus tract, and the other 55 patients were classified into the non-sinus tract group. The preoperative data, including basic demography, joint history (hip or knee, primary or revision) and infection types, were comparable between the two groups. The pathogen classification, rate of modular component exchanges and topical antibiotic administration were also similar between the two groups (Table 1).

The median follow-up time in all the patients was 4.4 (IQR: 2.05-7.91) years, and there was no significant difference between the two groups in follow-up time. At the last follow-up, 19 cases (36.5\%) had failed, and 3 cases $(5.8 \%)$ had died unrelated to PJI in the sinus tract group. Fifteen cases $(27.3 \%)$ had failed in the non-sinus tract group. The further treatment after the failed DAIR included suppressive antibiotic therapy, repeated DAIR, one-stage and two-stage exchange revision (Table 2). The cumulative infection control rate curve was depicted by the failure of DAIR as an end-point event, and the two groups showed similar cumulative infection control survivorship in the Kaplan-Meier curve (Fig. 1, $P=0.214$ ). The 1-year and 5-year cumulative infection control rates were $71.2 \%$ (95\% confidence interval (CI): $59.8-84.6 \%$ ) and $56.8 \%$ (95\% CI: 42.6-75.7\%) in the sinus tract group, respectively, while those in the non-sinus tract group were $83.6 \%$ (95\% CI: 74.4-94.0\%) and 71.3\% (95\% CI: 59.9-84.9\%), respectively.

Among the patients with a sinus tract, the successful group showed a higher proportion of modular component exchanges than the failed group ( $75.8 \%$ vs. $47.4 \%$, $P=0.038$ ) (Table 3). Patients after modular component exchange showed better infection control survivorship than patients without modular component exchange at the very edge of significance (Fig. $2, P=0.071$ ). Furtherly, we depicted the Kaplan-Meier curve among the patients without a sinus tract and the exchange of modular components did not influence the infection control survivorship among patients without a sinus tract (Fig. 3, $P=0.671)$. 
Table 1 The comparison of preoperative and intraoperative data between the sinus tract group and non-sinus tract group

\begin{tabular}{|c|c|c|c|}
\hline Variables & $\begin{array}{l}\text { Sinus tract } \\
(n=52)\end{array}$ & Non-sinus tract $(n=55)$ & P-Value \\
\hline Age, yrs. (mean $\pm S D)$ & $59.46 \pm 16.93$ & $63.48 \pm 11.64$ & $0.085^{\mathrm{a}}$ \\
\hline Male, n (\%) & $26(50.0 \%)$ & $19(34.5 \%)$ & $0.106^{b}$ \\
\hline $\mathrm{BMI}, \mathrm{kg} / \mathrm{m}^{2}($ mean $\pm \mathrm{SD})$ & $26.29 \pm 5.63$ & $26.82 \pm 4.17$ & $0.581^{\mathrm{a}}$ \\
\hline Median CCl, (IQR) & $3(2,4)$ & $3(2,4)$ & $0.317^{c}$ \\
\hline Joint (Hip), n (\%) & $17(32.7 \%)$ & $12(21.8 \%)$ & $0.206^{b}$ \\
\hline Revision, n (\%) & $12(23.1)$ & $8(14.5)$ & $0.258^{b}$ \\
\hline PJl type, n (\%) & & & $0.182^{b}$ \\
\hline Early PJI & $11(21.2 \%)$ & $14(25.5 \%)$ & \\
\hline Acute hematogenous PJI & $16(30.8 \%)$ & $24(43.6 \%)$ & \\
\hline Chronic PJI & $25(48.1 \%)$ & 17 (30.9\%) & \\
\hline Culture positive, n (\%) & $36(69.2 \%)$ & $36(65.5 \%)$ & $0.677^{b}$ \\
\hline Organism & & & $0.265^{d}$ \\
\hline Staphylococcus (MR) & $12(21.3 \%)$ & $10(18.2 \%)$ & \\
\hline Staphylococcus (MS) & $3(5.8 \%)$ & $5(9.1 \%)$ & \\
\hline Gram-negative & $6(11.5 \%)$ & $5(9.1 \%)$ & \\
\hline Other pathogens & $6(11.5 \%)$ & $13(23.6 \%)$ & \\
\hline Polymicrobial & $9(17.3 \%)$ & $3(5.5 \%)$ & \\
\hline Culture negative & $16(30.8 \%)$ & $19(34.5 \%)$ & \\
\hline Modular exchange, n (\%) & $34(65.4 \%)$ & $43(78.2 \%)$ & $0.141^{b}$ \\
\hline Topic antibiotics, n (\%) & $9(17.3 \%)$ & $12(21.8 \%)$ & $0.557^{b}$ \\
\hline Median follow-up, yrs. (IQR) & $3.18(1.80,7.68)$ & $5.38(2.42,7.98)$ & $0.182^{c}$ \\
\hline
\end{tabular}

$S D$ Standard deviation, $C C I$ Charlson Comorbidity Index, IQR Interquartile range, $P J /$ Periprosthetic joint infection, MR methicillin-resistant, $M S$ methicillin-sensitive; ${ }^{\text {a }}$ Student's T-test; ${ }^{b}$ Chi-squared test; ${ }^{C}$ Mann-Whitney U test; ${ }^{d}$ Fisher's exact test

Table 2 Further treatment after the failed debridement, antibiotics and implant retention (DAIR) in the sinus tract group and non-sinus tract group

\begin{tabular}{|c|c|c|c|c|}
\hline \multirow[t]{2}{*}{ Further treatment $^{a}$} & \multicolumn{2}{|c|}{ Sinus tract } & \multicolumn{2}{|c|}{ Non-sinus tract } \\
\hline & $\begin{array}{l}\text { Total } \\
(n=19)\end{array}$ & $\begin{array}{l}\text { Success }^{b} \\
7(36.8 \%)\end{array}$ & $\begin{array}{l}\text { Total } \\
(n=15)\end{array}$ & $\begin{array}{l}\text { Success }^{b} \\
10(66.7 \%)\end{array}$ \\
\hline $\begin{array}{l}\text { Suppressive antibiotic } \\
\text { therapy }\end{array}$ & $5(26.3 \%)$ & - & $3(20.0 \%)$ & - \\
\hline Multiple DAIR & $6(31.6 \%)$ & $3(50.0 \%)$ & $1(6.7 \%)$ & 1(100.0\%) \\
\hline $\begin{array}{l}\text { One-stage exchange } \\
\text { revision }\end{array}$ & $3(15.8 \%)$ & $2(66.7 \%)$ & $0(0.0 \%)$ & - \\
\hline $\begin{array}{l}\text { Two-stage exchange } \\
\text { revision }\end{array}$ & $5(26.3 \%)$ & $2(40.0 \%)^{c}$ & $11(73.3 \%)$ & $9(81.8 \%)$ \\
\hline
\end{tabular}

${ }^{a}$ Data are presented as numbers of patients (percentages); ${ }^{\mathrm{b}}$ The percentages indicate the proportion of patients who have infection control after the corresponding treatments; ${ }^{c}$ Another two patients have finished successful spacer implantation and are waiting for the two-stage revision

\section{Discussion}

As one of the major treatment options for PJI, the main concerns for DAIR is the unstable infection control rate in different studies [12]. Proper patient selection may help to ensure infection control rate after DAIR [13]. In this study, we first reported the 1-year and 5-year cumulative infection control rates (70.6 and 56.3\%, respectively) after DAIR for PJI in patients with a sinus tract and found that they were similar to their counterparts in patients without a sinus tract. More importantly, modular component exchange could improve the infection control rate in patients with a sinus tract.

Compared with one-stage and two-stage revision, DAIR avoids the significant challenges in removing the fixed prostheses, making joint reconstruction and increasing the risk of perioperative and postoperative complications [34]. The other advantages of DAIR include lower cost and better postoperative joint functions. As estimated, the cost in a two-stage revision is 2-3 times that in a DAIR case $[9,35]$. In the postoperative comparison between different PJI treatment options and non-infected primary arthroplasty, patients after successful DAIR share similar postoperative SF-12, Harris hip score and Western Ontario and McMaster Universities Osteoarthritis Index with patients after non-infected primary joint arthroplasty, while two-stage revision could not achieve comparable joint function scores $[7,8]$. These advantages of DAIR are appealing for most orthopedists and patients. Data from the Norwegian Arthroplasty Register showed that the annual number of DAIR cases for PJI after total knee arthroplasty and total hip arthroplasty has exceeded the total number of one-stage and 


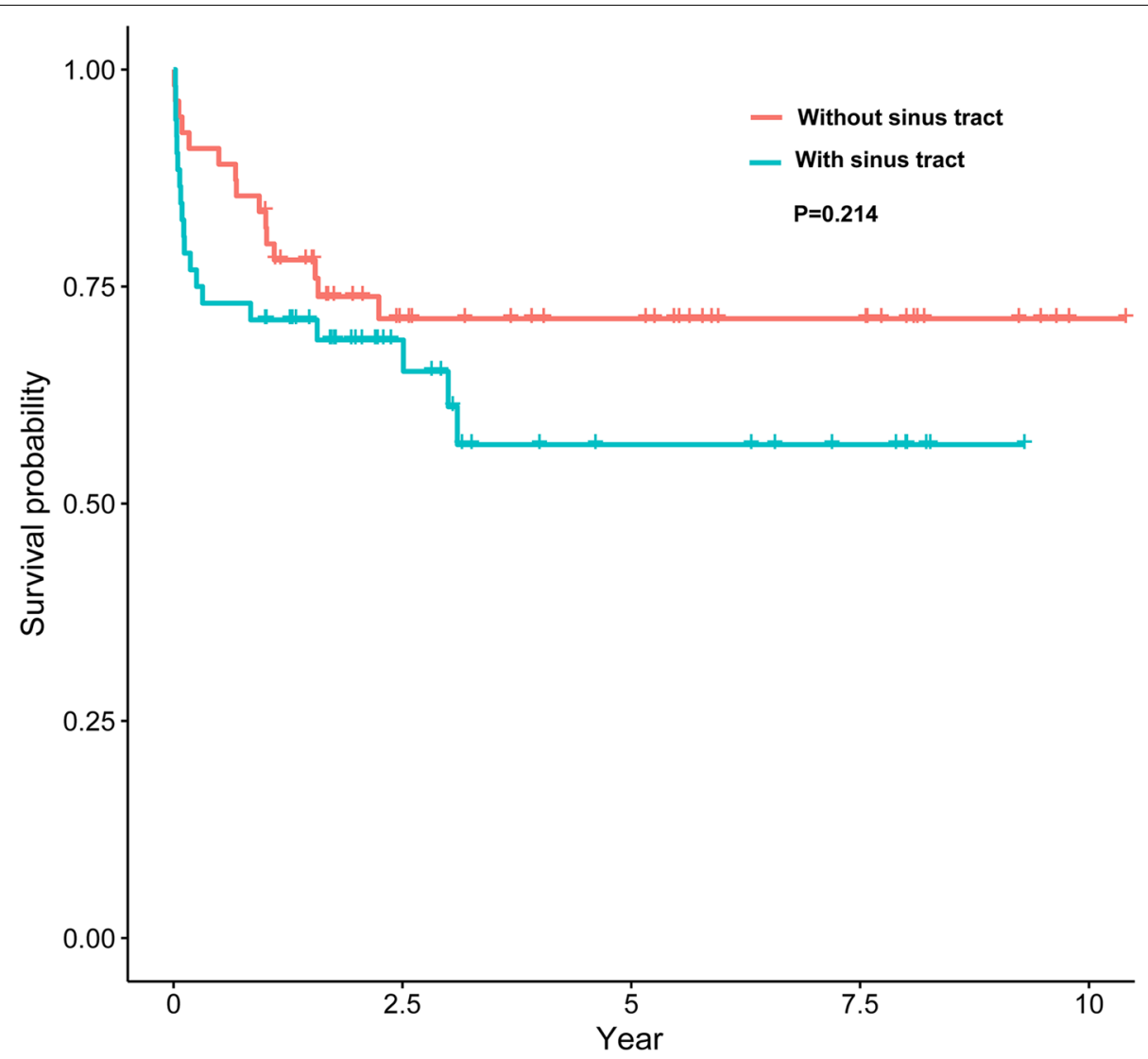

Fig. 1 The Kaplan-Meier survival curves for DAIR comparing the sinus tract group and non-sinus tract group

Table 3 The comparison of preoperative and intraoperative data between the successful and failed patients in the sinus tract group

\begin{tabular}{|c|c|c|c|}
\hline Variables & $\begin{array}{l}\text { Success } \\
(n=33)\end{array}$ & $\begin{array}{l}\text { Failure } \\
(n=19)\end{array}$ & P-Value \\
\hline Age, yrs. (mean $\pm S D)$ & $62.76 \pm 14.03$ & $53.74 \pm 20.19$ & $0.096^{\mathrm{a}}$ \\
\hline Male, n (\%) & $15(45.5 \%)$ & $11(57.9 \%)$ & $0.746^{b}$ \\
\hline $\mathrm{BMI}, \mathrm{kg} / \mathrm{m}^{2}($ mean $\pm \mathrm{SD})$ & $25.43 \pm 5.78$ & $27.79 \pm 5.17$ & $0.148^{\mathrm{a}}$ \\
\hline Median CCl, (IQR) & $3(2,4)$ & $2(1,4)$ & $0.224^{c}$ \\
\hline Joint (Hip), n (\%) & $9(27.3 \%)$ & $8(42.1 \%)$ & $0.272^{b}$ \\
\hline Revision, n (\%) & $7(21.2 \%)$ & $5(26.3 \%)$ & $0.937^{d}$ \\
\hline PJl type, n (\%) & & & $0.490^{\mathrm{b}}$ \\
\hline Early PJI & $6(18.2 \%)$ & $5(26.3 \%)$ & \\
\hline Acute hematogenous PJI & $12(36.4 \%)$ & $4(21.1 \%)$ & \\
\hline Chronic PJI & $15(45.5 \%)$ & $10(52.6 \%)$ & \\
\hline Culture positive, n (\%) & $24(72.7 \%)$ & $12(63.2 \%)$ & $0.472^{b}$ \\
\hline Modular exchange, n (\%) & $25(75.8 \%)$ & $9(47.4 \%)$ & $0.038^{b}$ \\
\hline Topic antibiotics, n (\%) & $4(12.1 \%)$ & $5(26.3 \%)$ & $0.356^{d}$ \\
\hline
\end{tabular}

SD: Standard deviation; CCI: Charlson Comorbidity Index; IQR: Interquartile range; PJl: Periprosthetic joint infection; ${ }^{a}$ Student's T-test; ${ }^{b}$ Chi-squared test; ${ }^{c}$ Mann-Whitney U test; ${ }^{\mathrm{d}}$ Continuity correction two-stage revisions in recent years [6, 10]. Expansion of DAIR in the treatment of PJI is in progress [25], and exploration of the possible risk factors for failure is of great importance.

The presence of a sinus tract was once recognized as a risk factor for failure after DAIR [28]. However, among all the previous publications that explored the potential risk factors for failure after DAIR, only the study from Marculescu et al. showed that sinus tracts could statistically increase the failure risk (Table 4) [15, 23-27, 36, 37]. Five studies did not reach a significant difference $[15,25-27,36]$, while two studies reported a lower failure rate in patients with a sinus tract $[23,24]$. Whether the presence of a sinus tract should be regarded as a risk factor is still a matter of debate. One of the possible reasons why only the study from Marculescu et al. reached a significant difference is the time of DAIR treatment. The cases reported by Marculescu et al. were performed during 1995-1999, while most DAIR cases included in the other seven studies were performed after 2000 [37]. In a recent meta-analysis, Kunutsor et al. reported that the overall infection control rate of DAIR performed before 2000 was only $51.50 \%$, which was lower than that 


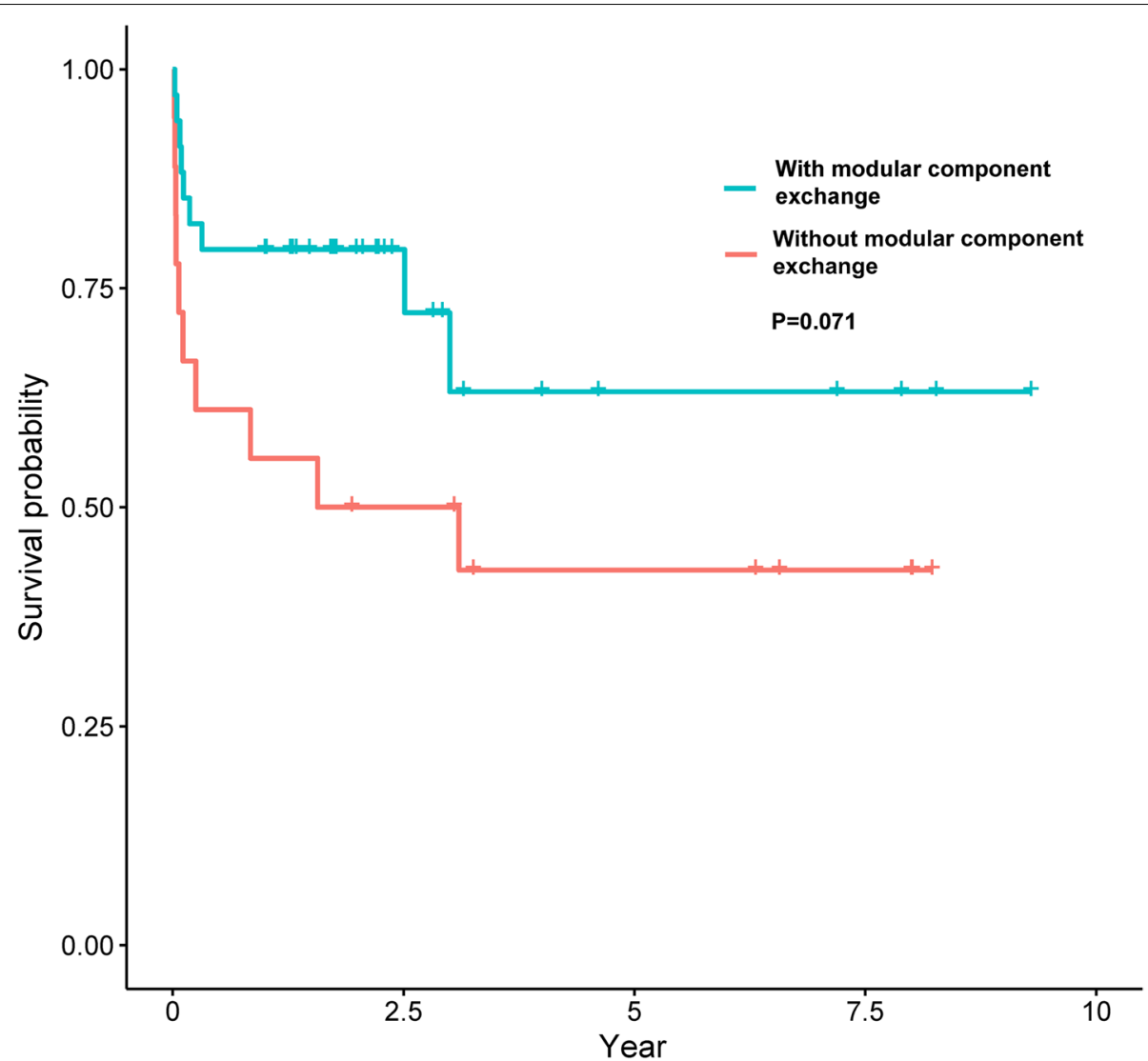

Fig. 2 The Kaplan-Meier survival curves for DAIR with or without exchange of modular component in the sinus tract group

of DAIR performed after $2000(61.2 \%, P=0.028)$ [12]. This improvement may be due to the amelioration of surgical techniques and the optimization of antibiotic administration $[12,18]$. The increase in the infection control rate after DAIR with time may have resulted in a better chance for infection control in patients with a sinus tract. In our study, all DAIRs were performed from 2009 to 2019, and the overall infection control rate was $67.3 \%$, which was slightly better than the data provided in Kunutsor et al's meta-analysis (61.2\%) [12]. Over 70\% of patients with a sinus tract could achieve infection control at the 1-year follow-up and over half at the 5-year followup. Anti-infection agents were recommended in debridement even without specific protocols available [13]. In our debridement process, we used repeated anti-infection agents ( $3 \%$ hydrogen peroxide and diluted iodophor solutions) in surgery according to the experience from traumatology, which may also contribute to the acceptable infection control rate in patients with a sinus tract.

The presence of a sinus tract has been pathognomonic for deep infection throughout surgical history and is recommended as one of the major criteria for the diagnosis of PJI [14, 38]. Although the surgical management of sinus tracts has been widely discussed, few studies have provided a detailed pathologic description of sinus tracts in PJI. A sinus tract could present in either acute or chronic infection, or evolve from prolonged postoperative drainage [14-16]. A recent prospective observational cohort study of 783 PJI patients from 27 centers in Australia and New Zealand reported that sinus tracts existed among 46 (23.4\%) of the 196 early PJIs [16]. Mu et al. also reported 73 patients treated with DAIR and topical antibiotics delivery for PJI within 3 months after the primary joint arthroplasty and the presence of sinus tract was high to $41 \%$ [15]. Even though this proportion is lower than that in chronic PJI (47.9\%), it still suggests that a sinus tract do not necessarily indicate chronic infection. $\mathrm{Xu}$ et al. reported five risk factors for the presence of a sinus tract, which included smoking $(\mathrm{OR}=1.83)$, hypothyroidism $(\mathrm{OR}=1.62)$, hypoalbuminemia $(\mathrm{OR}=1.52)$, hip joint involvement $(\mathrm{OR}=1.43)$, and prior revision surgery $(\mathrm{OR}=1.37)$ [39]. To our knowledge, no previous study reported specific risk factors for failure after DAIR in patients with a sinus tract.

In the exploration of possible risk factors for failure after DAIR among patients with a sinus tract, we 


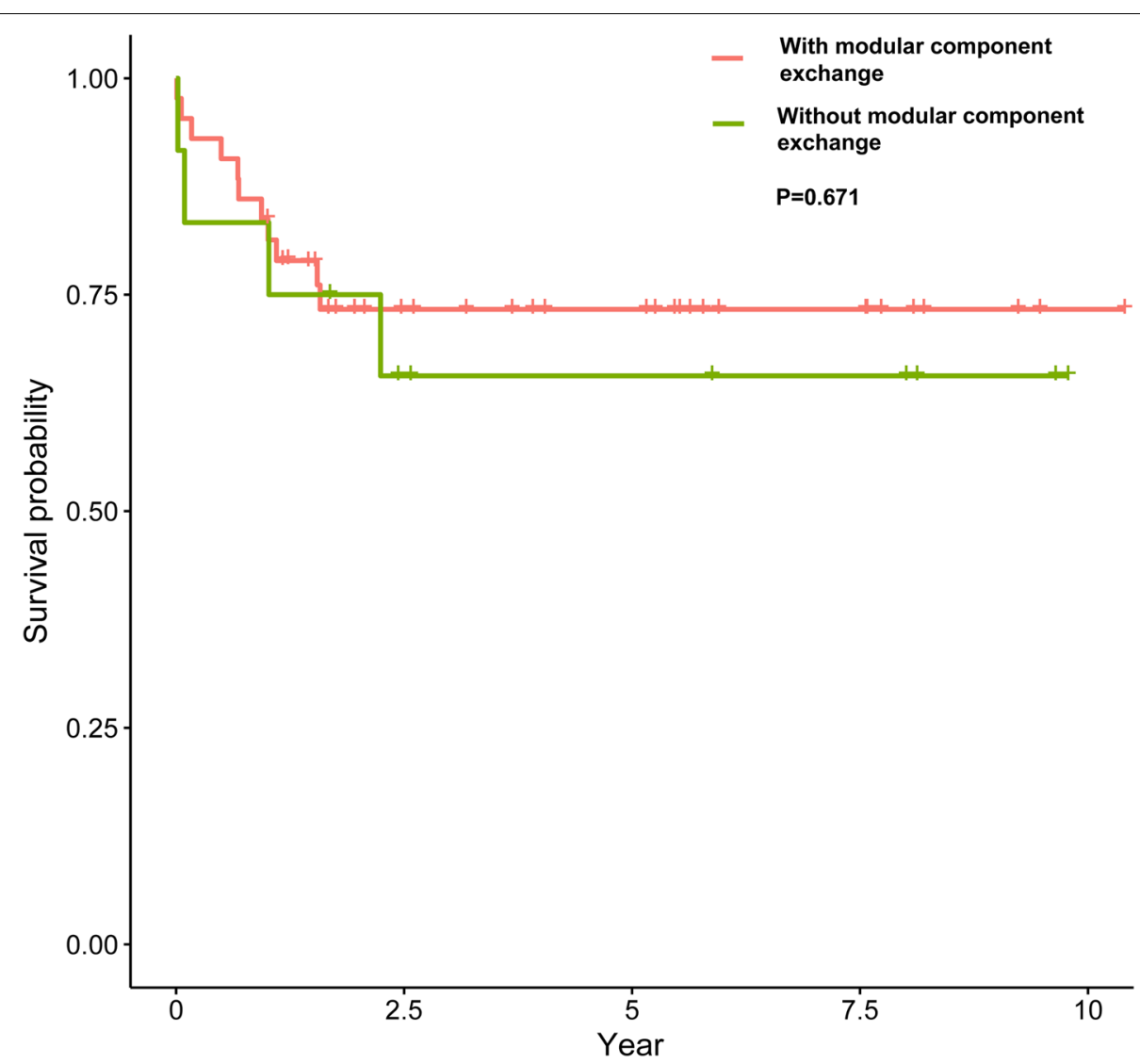

Fig. 3 The Kaplan-Meier survival curves for DAIR with or without exchange of modular component in the non-sinus tract group

Table 4 The comparison of success rate between the sinus tract group and non-sinus tract group in previous studies

\begin{tabular}{|c|c|c|c|c|c|c|c|c|}
\hline \multirow[t]{2}{*}{ Study } & \multirow[t]{2}{*}{ Centers/Nations } & \multirow[t]{2}{*}{ DAIR time } & \multicolumn{2}{|c|}{ Sinus tract $\mathbf{N}(\%)$} & \multicolumn{2}{|c|}{$\begin{array}{l}\text { Non-sinus tract } \\
\mathrm{N}(\%)\end{array}$} & \multirow[t]{2}{*}{ HR or OR $(95 \% \mathrm{Cl})$} & \multirow[t]{2}{*}{ P-value } \\
\hline & & & Success & Failure & Success & Failure & & \\
\hline $\begin{array}{l}\text { Mu et al. } \\
2020[15]\end{array}$ & Single center from China & 2011-2018 & $30^{\mathrm{a}}$ & & $43^{\mathrm{a}}$ & & $0.319(0.062-1.631)^{c}$ & 0.170 \\
\hline $\begin{array}{l}\text { Shohat et al. } \\
2020[23]\end{array}$ & $\begin{array}{l}27 \text { centers throughout the USA and } \\
\text { Europe }\end{array}$ & 2005-2017 & $212(70.2)$ & $90(29.8)$ & $557(63.9)$ & $315(36.1)$ & - & $0.049^{d}$ \\
\hline $\begin{array}{l}\text { Lesens et al. } \\
2018[25]\end{array}$ & 6 centers from France & 2010-2014 & $39^{\mathrm{a}}$ & & $98^{\mathrm{a}}$ & & $1.8(0.92-3.81)^{b}$ & 0.079 \\
\hline $\begin{array}{l}\text { Lowik et al. } \\
2018[24]\end{array}$ & 3 centers from Netherlands & $2006-2016$ & $32(78.0)$ & $9(22.0)$ & $206(59.7)$ & $139(40.3)$ & $0.42(0.19-0.90)^{c}$ & 0.022 \\
\hline $\begin{array}{l}\text { Lora-Tamayo et al. } \\
2017 \text { [26] }\end{array}$ & $\begin{array}{l}52 \text { centers throughout } \\
15 \text { nations }\end{array}$ & 2003-2012 & $34(55.7)$ & $27(44.3)$ & $223(59.0)$ & $155(41.0)$ & $1.12(0.75-1.69)^{b}$ & 0.58 \\
\hline $\begin{array}{l}\text { Tornero et al. } \\
2015 \text { [27] }\end{array}$ & Database from Spain & $1999-2014$ & $12(70.6)$ & $5(29.4)$ & $73(76.0)$ & $23(24.0)$ & $1.32(0.42-4.15)^{c}$ & 0.761 \\
\hline $\begin{array}{l}\text { Betz et al. } \\
2015[36]\end{array}$ & Single center from Switzerland & $1996-2012$ & $2(100)$ & $0(0)$ & 29 (80.6) & $7(19.4)$ & 0 & 1.000 \\
\hline $\begin{array}{l}\text { Marculescu et al. } \\
2006[37]\end{array}$ & Single center from USA & 1995-1999 & $15^{\mathrm{a}}$ & & $84^{\mathrm{a}}$ & & $2.85(1.50-5.44)^{b}$ & 0.002 \\
\hline
\end{tabular}

${ }^{\mathrm{a}}$ Exact failure number in each group was not available in the corresponding literature; ${ }^{\mathrm{b}} \mathrm{HR} ;{ }^{\mathrm{c}} \mathrm{OR} ;{ }^{\mathrm{d}} \mathrm{Chi}$-squared test 
noticed that modular component exchange could improve the PJI control rate at the last follow-up $(75.8 \%$ vs. $47.4 \%, P=0.038$ ) and showed better infectionfree survivorship in Kaplan-Meier survival analysis with a strong tendency towards statistical significance $(P=0.071)$. Modular component exchange was performed in nearly $72 \%$ of DAIR cases in the current study, which was similar to the national survey results that indicated that 74 of 99 institutions (75\%) performed modular component exchange for hip PJI and $82 \%$ for knee PJI in the Netherlands [40]. Modular component exchange has been recommended in ICM consensus after reviewing the present literature and available evidence $[13,28]$. In the failure predictive score reported by Wouthuyzen-Bakker et al., modular component exchange is also included as a protective factor [41]. Removing the modular components allows for better visualization and access of the articular cavity, especially in the posterior or deep aspect, and a larger working space, thereby facilitating more thorough debridement. At the same time, the exchange of modular components could directly reduce the bioburden and further help to eliminate the PJI. However, the exchange of modular components may be impeded by the lack of component supply in clinical practice and can increase the cost. DAIR without modular component exchange in patients with a sinus tract should be avoided.

This study has several limitations. First, as a retrospective cohort study, some inherent constraints may be embedded. However, it is impractical to perform studies with higher evidence to compare the infection control rates in patients with or without sinus due to the low incidence and urgent patient conditions. Second, the limitation of patient sample size may impede the detection of a significant difference in the comparison of cumulative infection-control survivorship between the two groups. However, useful information about the cumulative infection control rate in each group could also be provided by the Kaplan-Meier curve. Third, the pathogenic microorganisms were different between patients, as were the debridement skills among surgeons. Antibiotic administration may not be consistent in each patient. However, all these patients shared the same therapeutic protocol. Fourth, the follow-up time for some of the patients has not yet reached 5 years, the middle-term follow-up time set by the consensus meeting, meaning that some patients may experience failure in the future [42]. Longer follow-ups should be performed, and more patients should be included in future studies. Finally, since this study only focused patients after DAIR and did not compare them with patients after one-stage or two-stage revision, more future research still needs to be performed for the exploration of the best treatment options for PJI.

\section{Conclusions}

The presence of a sinus tract does not affect the success rate of DAIR. Modular component exchange in DAIR was proposed for patients with a sinus tract for an improved infection control rate.

\section{Abbreviations \\ DAIR: Debridement, antibiotics and implant retention (DAIR); PJI: Periprosthetic joint infection; ICM: International Consensus Meeting.}

\section{Acknowledgements}

We thank Zhaolun Wang and Hua Li for their assistance in the statistical analysis.

\section{Authors' contributions}

Study design: Wang Deng and Rui Li. Data collection/validation: Wang Deng and Baozhan Yu. Data analysis: Hongyi Shao and Rui Li. Result interpretation: Wang Deng and Rui Li. Reporting and editing: Wang Deng and Rui Li. Final approval of the version to be submitted: Jiying Chen and Yixin Zhou. Project guarantor: Jiying Chen and Yixin Zhou. Wang Deng and Rui Li contributed equally to this manuscript and are co-first authors. Jiying Chen and Yixin Zhou are the corresponding authors for this multi-center research. All authors have read and approved the manuscript.

\section{Authors' information}

Wang Deng, Hongyi Shao and Yixin Zhou come from Department of Orthopaedic Surgery, Beijing Jishuitan Hospital, Fourth Clinical College of Peking University, Beijing, China. Rui Li and Jiying Chen come from Department of Orthopaedics, Chinese PLA General Hospital, Beijing, China. Baozhan Yu comes from Department of Orthopaedics, Bao Ding Gem Flower Eastern Hospital, Bao Ding, Hebei, China.

\section{Funding}

This work was supported by Beijing Municipal Administration of Hospitals'. Ascent Plan (DFL20190401).

\section{Availability of data and materials}

The data that support the findings of this study are available from the corresponding authors but restrictions apply to the availability of these data, which were used under license for the current study, and so are not publicly available. Data are however available from the authors upon reasonable request and with permission of the hospital ethical institutional review board.

\section{Declarations}

\section{Ethics approval and consent to participate}

This study has been approved by our hospital ethical institutional review board. Informed consent was waived since this is a retrospective study with prerecorded database. This study was performed in line with the principles of the Declaration of Helsinki.

\section{Consent for publication}

Not applicable.

\section{Competing interests}

The authors declare that they have no competing interests.

\section{Author details}

${ }^{1}$ Department of Orthopedic Surgery, Beijing Jishuitan Hospital, Fourth Clinical College of Peking University, No.31 Xinjiekou East Road, Xicheng District, Beijing 100035, China. ${ }^{2}$ Department of Orthopaedics, Chinese PLA General Hospital, No. 28 Fuxing Road, Haidian District, Beijing 100039, China. 
${ }^{3}$ Department of Orthopaedics, Bao Ding Gem Flower Eastern Hospital, Bao Ding, Hebei, China.

Received: 7 August 2021 Accepted: 29 September 2021

Published online: 21 October 2021

\section{References}

1. Koh CK, Zeng I, Ravi S, Zhu M, Vince KG, Young SW. Periprosthetic joint infection is the Main cause of failure for modern knee arthroplasty: an analysis of 11,134 knees. Clin Orthop Relat Res. 2017;475(9):2194-201.

2. Kurtz SM, Lau EC, Son MS, Chang ET, Zimmerli W, Parvizi J. Are we winning or losing the Battle with Periprosthetic joint infection: trends in Periprosthetic joint infection and mortality risk for the Medicare population. J Arthroplast. 2018:33(10):3238-45.

3. Jonas K, Nils W, Alexander D, Stefan B, Henning W, Thilo F. The etiology of revision total hip arthroplasty: current trends in a retrospective survey of 3450 cases. Arch Orthop Trauma Surg. 2020;140(9):1265-73.

4. Kapadia BH, Banerjee S, Cherian JJ, Bozic KJ, Mont MA. The economic impact of Periprosthetic infections after Total hip arthroplasty at a specialized tertiary-care center. J Arthroplast. 2016;31 (7):1422-6.

5. Koh IJ, Han SB, In Y, Oh KJ, Lee DH, Kim TK. Open debridement and prosthesis retention is a viable treatment option for acute periprosthetic joint infection after total knee arthroplasty. Arch Orthop Trauma Surg. 2015;135(6):847-55.

6. Leta TH, Lygre SHL, Schrama JC, Hallan G, Gjertsen JE, Dale H, et al. Outcome of revision surgery for infection after Total knee arthroplasty: results of 3 surgical strategies. JBJS Rev. 2019;7(6):e4.

7. Aboltins C, Dowsey M, Peel T, Lim WK, Choong P. Good quality of life outcomes after treatment of prosthetic joint infection with debridement and prosthesis retention. J Orthop Res. 2016;34(5):898-902.

8. Herman BV, Nyland M, Somerville L, MacDonald SJ, Lanting BA, Howard $J$ L. Functional outcomes of infected hip arthroplasty: a comparison of different surgical treatment options. Hip Int. 2017;27(3):245-50.

9. Puhto T, Puhto AP, Vielma M, Syrjala H. Infection triples the cost of a primary joint arthroplasty. Infect Dis (Lond). 2019;51 (5):348-55.

10. Engesaeter LB, Dale H, Schrama JC, Hallan G, Lie SA. Surgical procedures in the treatment of 784 infected THAs reported to the Norwegian arthroplasty register. Acta Orthop. 2011;82(5):530-7.

11. Boyle KK, Kapadia M, Landy DC, Henry MW, Miller AO, Westrich GH. Utilization of debridement, antibiotics, and implant retention for infection after Total joint arthroplasty over a decade in the United States. J Arthroplast. 2020;35(8):2210-6.

12. Kunutsor SK, Beswick AD, Whitehouse MR, Wylde V, Blom AW. Debridement, antibiotics and implant retention for periprosthetic joint infections: a systematic review and meta-analysis of treatment outcomes. J Inf Secur. 2018;77(6):479-88.

13. Argenson JN, Arndt M, Babis G, Battenberg A, Budhiparama N, Catani $F$, et al. Hip and knee section, treatment, debridement and retention of implant: proceedings of international consensus on orthopedic infections. J Arthroplast. 2019;34(2S):S399-419.

14. Elkins JM, Kates S, Lange J, Lange J, Lichstein P, Otero J, et al. General assembly, diagnosis, definitions: proceedings of international consensus on orthopedic infections. J Arthroplast. 2019;34(2S):S181-5.

15. Mu W, Xu B, Guo W, Ji B, Wahafu T, Cao L. Outcome of irrigation and debridement with topical antibiotics delivery for the Management of Periprosthetic Joint Infection Occurred within 3 months since the primary Total joint arthroplasty. J Arthroplast. 2021;36(5):1765-71.

16. Manning L, Metcalf S, Clark B, Robinson JO, Huggan P, Luey C, et al. Clinical characteristics, etiology, and initial management strategy of newly diagnosed Periprosthetic joint infection: a multicenter, prospective observational cohort study of 783 patients. Open forum. Infect Dis. 2020;7(5):ofaa068.

17. Sendi P, Lotscher PO, Kessler B, Graber P, Zimmerli W, Clauss M. Debridement and implant retention in the management of hip periprosthetic joint infection: outcomes following guided and rapid treatment at a single Centre. Bone Joint J. 2017;99-B(3):330-6.

18. Ottesen CS, Troelsen A, Sandholdt H, Jacobsen S, Husted H, Gromov K. Acceptable success rate in patients with Periprosthetic knee joint infection treated with debridement, antibiotics, and implant retention. J Arthroplast. 2019;34(2):365-8.

19. Urish KL, Bullock AG, Kreger AM, Shah NB, Jeong K, Rothenberger SD. A multicenter study of irrigation and debridement in Total knee arthroplasty Periprosthetic joint infection: treatment failure is high. J Arthroplast. 2018;33(4):1154-9.

20. Shohat N, Goswami K, Tan TL, Fillingham Y, Parvizi J. Increased failure after irrigation and debridement for acute Hematogenous Periprosthetic joint infection. J Bone Joint Surg Am. 2019;101(8):696-703.

21. Qu GX, Zhang CH, Yan SG, Cai XZ. Debridement, antibiotics, and implant retention for periprosthetic knee infections: a pooling analysis of 1266 cases. J Orthop Surg Res. 2019;14(1):358.

22. Tsang SJ, Ting J, Simpson A, Gaston P. Outcomes following debridement, antibiotics and implant retention in the management of periprosthetic infections of the hip: a review of cohort studies. Bone Joint J. 2017;99-B(11):1458-66.

23. Shohat N, Goswami K, Tan TL, Yayac M, Soriano A, Sousa R, et al. 2020 frank Stinchfield award: identifying who will fail following irrigation and debridement for prosthetic joint infection. Bone Joint J. 2020;102-B(7_Supple_B):11-9.

24. Lowik CAM, Jutte PC, Tornero E, Ploegmakers JJW, Knobben BAS, deVries $\mathrm{AJ}$, et al. Predicting failure in early acute prosthetic joint infection treated with debridement, antibiotics, and implant retention: external validation of the KLIC score. J Arthroplast. 2018;33(8):2582-7.

25. Lesens O, Ferry T, Forestier E, Botelho-Nevers E, Pavese P, Piet E, et al. Should we expand the indications for the DAIR (debridement, antibiotic therapy, and implant retention) procedure for Staphylococcus aureus prosthetic joint infections? A multicenter retrospective study. Eur J Clin Microbiol Infect Dis. 2018;37(10):1949-56.

26. Lora-Tamayo J, Senneville E, Ribera A, Bernard L, Dupon M, Zeller V, et al. The not-so-good prognosis of streptococcal Periprosthetic joint infection managed by implant retention: the results of a large multicenter study. Clin Infect Dis. 2017;64(12):1742-52.

27. Tornero E, Morata L, Martinez-Pastor JC, Bori G, Climent C, Garcia-Velez $\mathrm{DM}$, et al. KLIC-score for predicting early failure in prosthetic joint infections treated with debridement, implant retention and antibiotics. Clin Microbiol Infect. 2015;21(8):786 e9-e17.

28. Parvizi J, Gehrke T, Chen AF. Proceedings of the international consensus on Periprosthetic joint infection. Bone Joint J. 2013;95-B(11):1450-2.

29. Tsukayama DT, Goldberg VM, Kyle R. Diagnosis and management of infection after total knee arthroplasty. J Bone Joint Surg Am. 2003;85-A Suppl 1:S75-80.

30. Kim YH, Park JW, Kim JS, Kim DJ. The outcome of infected total knee arthroplasty: culture-positive versus culture-negative. Arch Orthop Trauma Surg. 2015;135(10):1459-67.

31. Moran E, Masters S, Berendt AR, McLardy-Smith P, Byren I, Atkins BL. Guiding empirical antibiotic therapy in orthopaedics: the microbiology of prosthetic joint infection managed by debridement, irrigation and prosthesis retention. J Inf Secur. 2007;55(1):1-7.

32. Bernard L, Legout L, Zurcher-Pfund L, Stern R, Rohner P, Peter R, et al. Six weeks of antibiotic treatment is sufficient following surgery for septic arthroplasty. J Inf Secur. 2010;61 (2):125-32.

33. Diaz-Ledezma C, Higuera CA, Parvizi J. Success after treatment of periprosthetic joint infection: a Delphi-based international multidisciplinary consensus. Clin Orthop Relat Res. 2013;471(7):2374-82.

34. Qasim SN, Swann A, Ashford R. The DAIR (debridement, antibiotics and implant retention) procedure for infected total knee replacement - a literature review. SICOT J. 2017;3:2.

35. Sousa A, Carvalho A, Pereira C, ReisE SAC, Abreu M, et al. Economic impact of prosthetic joint infection - an evaluation within the Portuguese National Health System. J Bone Jt Infect. 2018;3(4):197-202.

36. Betz M, Abrassart S, Vaudaux P, Gjika E, Schindler M, Billieres J, et al. Increased risk of joint failure in hip prostheses infected with Staphylococcus aureus treated with debridement, antibiotics and implant retention compared to Streptococcus. Int Orthop. 2015;39(3):397-401.

37. Marculescu CE, Berbari EF, Hanssen AD, Steckelberg JM, Harmsen SW, Mandrekar JN, et al. Outcome of prosthetic joint infections treated with debridement and retention of components. Clin Infect Dis. 2006;42(4):471-8.

38. Amanatullah D, Dennis D, Oltra EG, Marcelino Gomes LS, Goodman SB, Hamlin B, et al. hip and knee section, diagnosis, definitions: proceedings 
of international consensus on orthopedic infections. J Arthroplast. 2019;34(2S):S329-37.

39. Xu C, Wang Q, Kuo FC, Goswami K, Tan TL, Parvizi J. The presence of sinus tract adversely affects the outcome of treatment of Periprosthetic joint infections. J Arthroplast. 2019;34(6):1227-1232.e2.

40. Veltman ES, Moojen DJF, Nelissen RG, Poolman RW. Antibiotic prophylaxis and DAIR treatment in primary Total hip and knee arthroplasty, a National Survey in the Netherlands. J Bone Jt Infect. 2018;3(1):5-9.

41. Wouthuyzen-Bakker M, Sebillotte M, Lomas J, Taylor A, Palomares EB, Murillo $\mathrm{O}$, et al. Clinical outcome and risk factors for failure in late acute prosthetic joint infections treated with debridement and implant retention. J Inf Secur. 2019;78(1):40-7.

42. Abblitt WP, Ascione T, Bini S, Bori G, Brekke AC, Chen AF, et al. Hip and knee section, outcomes: proceedings of international consensus on orthopedic infections. J Arthroplast. 2019;34(2s):S487-95.

\section{Publisher's Note}

Springer Nature remains neutral with regard to jurisdictional claims in published maps and institutional affiliations.
Ready to submit your research? Choose BMC and benefit from:

- fast, convenient online submission

- thorough peer review by experienced researchers in your field

- rapid publication on acceptance

- support for research data, including large and complex data types

- gold Open Access which fosters wider collaboration and increased citations

- maximum visibility for your research: over $100 \mathrm{M}$ website views per year

At BMC, research is always in progress.

Learn more biomedcentral.com/submissions 